\title{
Imaging Tools for the Developmental Biologist: Ultrasound Biomicroscopy of Mouse Embryonic Development
}

\author{
COLIN KIT LUN PHOON
}

Pediatric Cardiology Program, New York University School of Medicine, New York, NY 10016

\begin{abstract}
Progress has been rapid in the elucidation of genes responsible for cardiac development. Strategies to ascertain phenotypes, however, have lagged behind advances in genomics, particularly in the in vivo mouse embryo, considered a model organism for mammalian development, and for human development and disease. Over the past several years, our laboratory and others have pioneered a variety of ultrasound biomicroscopy (UBM)-Doppler approaches to study in vivo development in both normal and mutant mouse embryos. This state-of-the-art review will discuss the development and potential of ultrasound biomicroscopy as a tool for the in vivo imaging and phenotyping of both cardiac and non-cardiac organ systems in the early developing mouse. Broad, long-term research objectives are to define living structure-function relationships during critical periods of mammalian morphogenesis. (Pediatr Res 60: 14-21, 2006)
\end{abstract}

$\mathrm{C}^{\circ}$ ongenital malformations are generally believed to result from developmental processes gone awry. Highly useful for defining mammalian gene function and for determining the functional consequences of gene expression, the mouse is currently the animal model of choice for studying human development and disease $(1,2)$. Genetically-engineered mice, particularly with cardiovascular defects, often die in embryonic and fetal life, similar to human beings (3). Therefore, detailed phenotypic evaluation of such models is critical to the understanding of disease development. However, technologies and approaches for analyzing mutant phenotypes have fallen behind our ability to manipulate the mouse genome. For suspected abnormalities in cardiovascular development, mouse phenotyping generally begins with determination of survival. Until relatively recently, subsequent structural phenotyping has relied on gross examination and histologic techniques, but these are cumbersome and permit only snapshot, post-mortem analyses of static structure.

In the study of developmental processes, desirable characteristics of imaging include: acquisition of in situ data in a non-destructive and non-invasive manner (for the mouse, this means in utero, sub-surface imaging); adequate spatial and temporal resolution; and definition of both structure and func-

Received November 2, 2005; accepted February 17, 2006.

Correspondence: Colin K.L. Phoon, M.Phil., M.D., NYU Pediatric Cardiology Program, 530 First Avenue, FPT Suite 9U, NY, NY 10016; e-mail: colin.phoon@ med.nyu.edu

This work was supported in part by a National Institutes of Health Research Career Award (K08 HL04414), and was presented in part at the 2005 Pediatric Academic Societies Annual Meeting, Washington, DC.

DOI: 10.1203/01.pdr.0000219441.28206.79 tion. Ultrasound's strengths make it a particularly attractive imaging modality (Table 1). Ultrasonic visualization of living tissue at microscopic resolution is currently known as ultrasound biomicroscopy (UBM) (4), and UBM has emerged as a key imaging tool for embryonic mouse research. This stateof-the-art review will discuss the development and potential of UBM as a tool for in vivo imaging and phenotyping of the early developing mouse. Because of our laboratory's research interests, we will focus on cardiovascular development and developmental physiology. Cognizant of the needs of the greater developmental biology community, we will also briefly review UBM imaging and phenotyping of non-cardiac organ systems.

\section{PHENOTYPING APPROACHES: AN OVERVIEW}

Phenotyping strategies must overcome the challenges of tiny organism size, imaging within a muscular uterus (if to be imaged in vivo), and large litters (imaging and following multiple embryos). Cardiovascular development has been particularly difficult to analyze because of a paucity of efficient methods to characterize in vivo hemodynamics and structures in mouse embryos (5). However, a battery of complementary imaging modalities has emerged over the past 5-10 y that has broadened our ability to phenotype the mouse embryo. To frame the utility of UBM in proper context, a brief overview of several important modalities is provided here; the reader is referred to a recent review on imaging of the developing pacemaking and cardiac conduction systems (6), which is beyond the scope of this article.

Whole embryo culture: imaging and phenotyping. Cultured mouse embryos appear to be viable with normal growth for 1-2 d despite disruption of the embryonal-placental circulation, allowing a variety of physiologic and structural phenotyping approaches under carefully controlled conditions. Various imaging and phenotyping modalities under culture conditions permit optical resolution, including both highspeed and time-lapse videography (7-9), and direct measure-

Abbreviations: E, embryonic day (post-conceptional day); $\boldsymbol{\mu M R I , ~ m a g n e t i c ~}$ resonance microimaging; NFAT, Nuclear Factor of Activated T-cells; UBM, ultrasound biomicroscopy 
Table 1. Strengths and weaknesses of ultrasound imaging

\begin{tabular}{ll}
\hline \multicolumn{1}{c}{ Strengths } & \multicolumn{1}{c}{ Weaknesses } \\
\hline Non-invasive & Images limited by gas, bone, depth \\
Safe & Anesthesia needed \\
Portable, versatile & Needs special expertise/training* \\
Dynamic, real-time imaging & Operator-dependent image acquisition, \\
& analysis \\
Quantitative information on & No information on biochemical, \\
structure, function & metabolic processes \\
Spatial resolution (UBM) & Limited depth of penetration and \\
& poor tissue contrast (UBM) \\
\hline
\end{tabular}

* Since ultrasound is the most widely-used cross-sectional imaging modality in the world (71), many people are already trained in the basic principles of ultrasound imaging, including researchers, cardiologists, perinatologists, and sonographers. Such a pool of potential imaging specialists contrasts with the pool available for microscopic high-speed videomicroscopy techniques and $\mu \mathrm{MRI}$, for example.

ment of intra-arterial and intra-cardiac pressures using invasive techniques is possible $(7,10,11)$. Notably, vascular development and erythrocyte flow dynamics can be imaged using transgenic fluorescent technologies with whole-embryo culture $(8,9)$.

Micro-magnetic resonance imaging ( $\mu M R I)$. Advantages of $\mu \mathrm{MRI}$ include its non-destructive nature, excellent tissue contrast, and ability to reconstruct images in any plane, including three-dimensional (3-D) reconstruction. High spatial resolution $(25-50 \mu \mathrm{m})$ with exquisite morphologic detail can be achieved, but for the most part requires field strengths of 7-11 $\mathrm{T}$ and long acquisition times (hours-typically overnight); moreover, technical considerations limit $\mu$ MRI largely to fixed embryos (12-14). Efficient, high-throughput imaging is only just dawning $(14,15)$. Investigators have attempted in utero imaging of mouse embryos with some success, but spatial resolution has been on the order of $200 \mu \mathrm{m}(16,17)$.

Optical coherence tomography (OCT). The optical equivalent of ultrasound, OCT possesses optical resolution and permits in vivo imaging (18). Due to its depth of penetration of only $\sim 2-3 \mathrm{~mm}$, however, it has been achieved for the developing chick and zebrafish cardiovascular systems, but cannot be applied to the mouse embryo enveloped in utero.

Optical projection tomography (OPT). OPT is well-suited to the scale of the mouse embryo and can produce images of remarkable clarity, particularly 3-D images. OPT's effective resolution is $5-10 \mu \mathrm{m}$. Additional major strengths include the ability to map gene expression patterns through the visualization of immunofluorescent staining; other vital stains commonly used in histology can be visualized as well. Currently, OPT is an in vitro imaging technique and cannot be used for living embryos $(19,20)$.

Ultrasound in developmental biology: clinical ultrasound systems. The physics of ultrasound and the many aspects that play into constructing a detailed, high-resolution image have been extensively reviewed $(4,21)$. Developmental processes, occurring in very small organisms, mandate high transducer frequencies to achieve adequate spatial resolution in both the axial and lateral axes. UBM transducers operate at 40-100 $\mathrm{MHz}$ center frequencies, in contrast to diagnostic clinical transducers, which typically use 2-15 MHz. Important imaging issues that arise with high frequencies include loss of penetration, loss of depth of field, and changes in the ultrasound backscatter from blood $(4,21,22)$. Temporal resolution is important for moving objects such as the rapidly beating embryonic heart (23-27), albeit less so for static structures such as non-cardiac organs. By detecting frequency shifts reflected from moving targets (i.e., blood cells), pulsed-wave Doppler permits the detection of flow velocities within a specified region of interest, or sample volume. The size of the sample volume and the frequency of the Doppler incident beam are important determinants of the capabilities for embryonic imaging; ideally, one would like a sample volume small enough to interrogate separate regions within the developing embryo, while allowing for the detection of flow velocities approximately one order of magnitude smaller than in human beings $(24,25)$. Despite the application of potentially damaging acoustical energy to living tissue, the evidence to date points to no significant, deleterious effects of UBM on the developing mouse embryo $(25,28,29)$.

Imaging of the in utero developing mouse embryo is a nascent field. Most ultrasound studies, including UBM studies, have focused on functional analysis of different mouse models, and in abnormal models, have revealed various patterns of abnormal hemodynamics (23,30-32). However, technological limitations are apparent in studies that have used clinical ultrasound systems, including "high frequency" (13-15 MHz) systems, especially at stages during critical periods of morphogenesis. For example, the resolution of 7.5-MHz clinical ultrasound systems has been inadequate to image specific embryonic structures or to localize abnormalities in blood flow precisely by Doppler $(23,30)$. Using a $14 \mathrm{MHz}$ transducer to assess morphology and size of E7.518.5 embryos, Chang et al. (33) detected a heartbeat first at E10.5, which is more than $48 \mathrm{~h}$ later than the true initiation of the heartbeat (26). Resolution of 13-15 MHz systems is insufficient to distinguish left from right sides of the heart even through E18.5 (near-term), and investigators cannot localize separate inflow and outflow tracts (34). In studies of ENUinduced mutagenesis, investigators have really only diagnosed cardiac malformations (excluding hypertrophy, ectopia cordis, and outflow tract regurgitation) at postnatal necropsy, not by ultrasound-in other words, not in the living embryo or fetus $(35,36)$. The poor performance of even $15 \mathrm{MHz}$ systems is due to axial resolution of $440 \mu \mathrm{m}$, with lateral resolution of $630 \mu \mathrm{m}$ (37); to provide an idea of scale, the diameter of the embryonic mouse dorsal aorta is in the range of $300 \mu \mathrm{m}(38)$. Despite the inadequate spatial resolution, however, clinical ultrasound systems possess color Doppler flow mapping, not currently available on UBM imaging systems, that may aid in screening for major cardiac malformations (36).

\section{UBM-DOPPLER INSTRUMENTATION AND PHYSICS}

Ultrasound biomicroscopy: filling the gap. UBM-Doppler has emerged as the most promising of imaging modalities for the embryonic mouse, given its high resolution $(30-40 \mu \mathrm{m}$ axial and $70-90 \mu \mathrm{m}$ lateral resolution) and capability for 
subsurface, in utero imaging of early mouse embryos $(25,39)$. Analogous to clinical imaging modalities, UBM-Doppler imaging modalities include cross-sectional or two-dimensional (B-mode) imaging; one-dimensional M-mode imaging; and spectral pulsed-wave Doppler. Color flow imaging may eventually be routinely possible with UBM, but has been difficult to attain for embryonic imaging due to limitations inherent in mechanical transducers, and the trade-off between scan speed and minimal detectable blood flow velocities for the frequencies and spatial resolutions needed (40). Advances in UBM instrumentation now permit the operator to control frequency and other settings (pulse repetition frequency, system bandwidth, etc.), to refine the spatial resolution and depth of penetration needed to phenotype various stages of development $(22,25)$.

UBM has a depth of penetration typically of only 5-15 mm; this range, however, is quite adequate for most in utero embryonic mouse work. Current transducer technology yields a relatively narrow zone of focus (depth of field), but the development of annular array transducers should increase the distance over which an image remains in focus (41). Tissue contrast tends to be worse than with $\mu$ MRI or OPT. At UBM frequencies, blood in the mouse embryo appears echo-dense (bright, as opposed to the dark, echo-lucent areas imaged in clinical medicine) thus reducing one's ability to discriminate boundaries $(4,25,27,28,42)$. The temporal resolution is generally better on clinical systems than on UBM systems, but the frame rates on UBM systems-initially $8-10 \mathrm{~Hz}$ (frames/s) $(24,25)$ - are improving $(43)$ and at $60-100 \mathrm{~Hz}$ using mechanical sector scanning, now approach those of clinical systems. Still higher frame rates await the development of phased array transducers (4). Strategies to slow the heart rate down, such as cooling the external environment, will lead to improved visualization of embryonic function and structures (28), but such maneuvers impair normal physiologic processes. Thus, among the challenges to imaging is the ability to glean morphologic and functional data from the mouse embryo in a physiologic milieu.

As with virtually all in vivo imaging approaches, anesthesia is required and may alter the behavior of embryonic hearts, as well as maternal hemodynamics (44-46). In adult mice, isoflurane appears to provide the most stable hemodynamics of any anesthetic agent, although it also demonstrates at least mild adverse systemic effects $(45,46)$. Data on anesthetic effects on embryonic and fetal cardiac function are scant, and on isoflurane on in utero cardiac function non-existent. In one study, tribromoethanol induced arrhythmias in mouse embryos, whose heart rates were lower than those under pentobarbital (47). Furthermore, data on teratogenic effects of isoflurane have shown that pregnancy rates and fetal survival do not appear significantly reduced $(48,49)$, so that it is unlikely that isoflurane induces severe cardiac malformations. However, there may be an increased incidence of anomalies and mild growth retardation $(49,50)$, and exposed preimplantation embryos exhibit arrested development (51). In most of these studies, the exposure time to isoflurane was in the range of 2-4 h daily, for several days through gestation, far longer than in imaging studies. Nevertheless, one consid- eration in the use of anesthesia is that mutant embryos with abnormal cardiovascular physiology may be more sensitive to effects of anesthesia than their littermate controls.

\section{MOUSE IN UTERO UBM-DOPPLER APPROACHES}

Advancement of the imaging approach is at least as important as development of the physical instrumentation. Our laboratory's innovative multi-parameter in utero approach has yielded insights into integrative cardiovascular physiology, accounting not only for heart function but also the interactions of the heart with different intra- and extra-embryonic vascular beds $(24,38,39)$. The developing embryo is exquisitely sensitive to environmental perturbations such as hypothermia, such that even non-physiological heart rates will change many hemodynamic parameters, such as contractile ability, relaxation, and regional blood flow $(10,52)$. We have therefore developed stringent protocols yielding physiologic in utero experimental conditions $(24,26,27)$. The excellent spatial resolution of UBM, coupled with precise Doppler sample volume placement, permits non-invasive characterization of blood flow parameters in the in utero mouse embryo not possible with clinical ultrasound systems (Fig. 1) (25,38); various functional UBM-Doppler parameters have recently been reviewed in detail elsewhere (39). This integrated approach to cardiovascular physiology, when combined with cellular and molecular techniques, is uniquely suited to answering many fundamental questions posed in developmental biology.

Two in utero imaging approaches have been developed. With traditional non-invasive (i.e., trans-abdominal or transcutaneous) imaging approach, embryos are imaged within the abdomen through the maternal abdominal wall (Fig. 2). Early UBM equipment consisted of separate imaging and Doppler transducers $(24,39,42)$, with mechanical motion of the imaging transducer precluding efficient non-invasive imaging, since it could not be moved easily over the abdominal surface

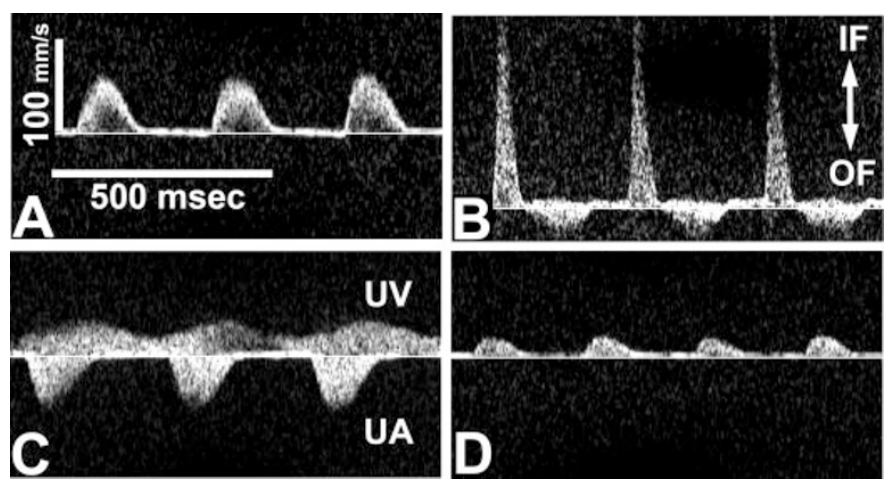

Figure 1. Multiple sites interrogated by pulsed-wave spectral UBM-Doppler in the same E12.5 embryo. $A$ ) dorsal aorta; $B$ ) inflow (IF-note the absence of an E-wave at this early developmental stage) and outflow (OF); C) umbilical artery (UA) and vein (UV); $D$ ) vitelline artery. Our experience and others' demonstrates that obtaining consistent inflow-outflow Doppler data remains inconsistent, due to the precision with which one must place and maintain the sample volume; indeed, although this inflow-outflow pattern was probably taken from the left ventricle, the small size of the heart and its motion likely mean that right ventricular inflow-outflow signal is contaminating this. The velocity $(100 \mathrm{~mm} / \mathrm{s})$ and time $(500 \mathrm{~ms})$ scales are equivalent for all panels. 

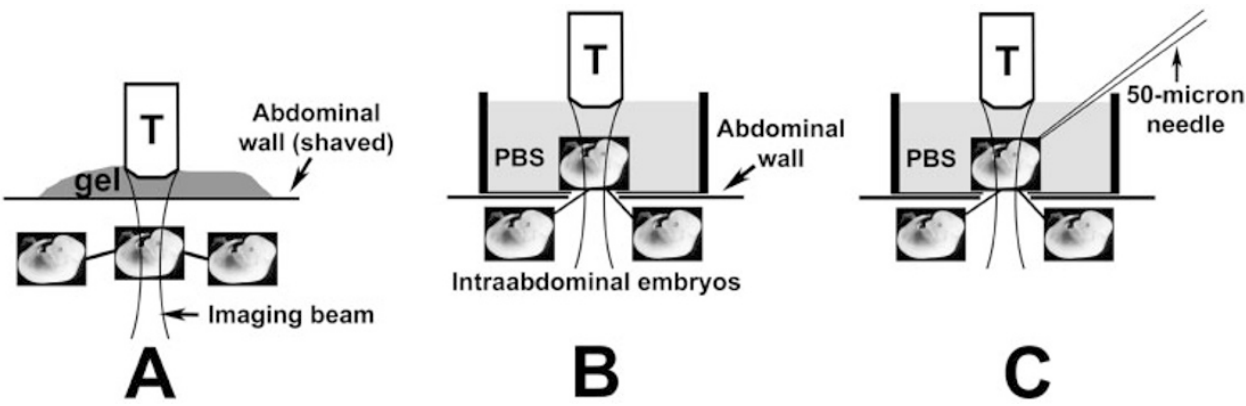

Figure 2. Schematic of UBM imaging approaches. A) Non-invasive approach: Intra-abdominal embryos are imaged through the shaved abdominal wall. $B$ ) Semi-invasive approach: Through a small vertical abdominal incision, one embryo, still encased within its uterine sac, is exteriorized into warmed PBS in a Petri dish. C) Micro-injection approach: The semi-invasive approach can be used to perform microinjections with a pulled $50 \mu \mathrm{m}$ glass needle, which is advanced through the uterine wall with a micromanipulator/injector. In all panels, the embryos are shown schematically connected to one another within their respective uterine sacs, and the imaging and Doppler transducers are contained within the single housing ( $\mathrm{T}$ ). As much fur must be removed from the skin as possible (by shaving or depilatory cream), since fur harbors air, reducing imaging effectiveness; centrifuging the ultrasound coupling gel may also help. In all experimental approaches, both the pregnant mouse and the acoustical coupling medium (gel or PBS bath) are maintained at a physiologic temperature of $37 \pm 1{ }^{\circ} \mathrm{C}$ to achieve physiologic embryonic temperatures $(24,27,43)$.

to locate and track multiple embryos. In early experiments, we obtained pooled data by imaging up to four embryos per litter, but without knowing exactly where they were within the litter (24). Therefore, genotype-phenotype correlation was not possible with this instrumentation.

To overcome such technological limitations, we developed a semi-invasive approach to UBM-Doppler imaging. A small incision is made in the abdominal wall, and one to two embryos, still encased within the uterine sac and in continuity with the litter, are gently exteriorized into warmed physiologic saline in a Petri dish (Fig. 2). This approach has yielded excellent physiologic imaging conditions and data $(26,27)$. Advantages to the semi-invasive imaging approach include the exclusion of the maternal abdominal wall and intraabdominal contents from the imaging beam path, leading to better resolution of embryonic structures $(25,53)$. This approach is also now serving us well in UBM-guided microinjection experiments (see below).

One recent development is the ability to image mouse embryos non-invasively and in longitudinal fashion using newer UBM transducers that contain the imaging and Doppler transducer within one housing. Although reported using 7.5-15 MHz clinical transducers in later-stage mouse embryos and fetuses $(31,32,35-37)$, serial UBM imaging remains difficult, particularly in early development (54). We recently reported our ability to localize $100 \%$ of living and recentlydead (non-resorbed) E10.5-17.5 embryos at any given time, as confirmed by wide laparotomy (43). Living and dead embryos were easily distinguishable from other intra-abdominal structures (Fig. 3). To simulate a cardiac screening assay, aortic and intra-cardiac Doppler flows, and UBM cardiac images were obtained in all living embryos, with accurate correlation of phenotype (reversed dorsal aortic flow) with genotype (NFATC1-/-). Embryos were also followed longitudinally at various stages from E12.5 through E16.5 for 1 to 2 d. The presence of marker embryos (e.g., resorbed embryos and ones with abnormal flows) facilitated the identification of individual embryos at follow up. Changes in cardiovascular physiology in individual embryos can now be determined as morphology and function evolve.
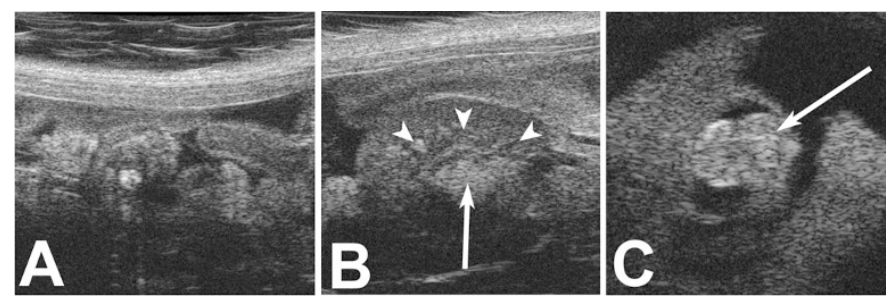

Figure 3. Living embryos are readily distinguished from other intraabdominal structures. A) Maternal bowel loops; $B$ ) maternal kidney (arrow indicates renal pelvis; arrowheads indicate medulla); $C$ ) dead embryo (arrow indicates a non-beating heart within a pericardial effusion).

\section{DEVELOPMENTAL CARDIOVASCULAR PHYSIOLOGY BY UBM-DOPPLER}

Initiation and maturation of mammalian circulation. Over the past several years, UBM-Doppler has facilitated detailed characterization of the embryonic mouse circulation from its earliest stages. Following reports of blood flow imaging at E8.5 $(25,42)$, precise staging from our laboratory (26) demonstrated rhythmic cardiac contractions as early as 5-somites, $\sim$ E8.25; blood flow was detectable by Doppler by the 7-8 somite stage. At these stages, the mouse heart is approximately a straight tube. This earliest cardiac functioning coincided precisely with the entry of primitive erythrocytes into the embryo proper, from the hematopoietic yolk sac. Thus, establishment and early development of the initial circulation are precisely coordinated to bring together a functioning pump, an intact vascular circuit, and oxygen-carrying erythrocytes to support the growing embryo. Notably, the earliest origins of a functional cardiovascular system occur well before the embryo needs convection of oxygen and nutrients $(26,55)$, but are likely necessary for cardiac morphogenesis and vasculogenesis (55).

Heart rate and systolic cardiac work subsequently increase to meet the metabolic demands of the growing embryo $(24,56)$, and Doppler indices indicate that placental vascular impedance falls, from even early in development (24). Unlike in human beings, the murine heart rate continues to rise 
postnatally as part of its developmental maturation (57). Diastolic cardiac function has been studied at mid- to late gestation, extending into the early postnatal and young adult stages. As characterized by the E/A waves across the atrioventricular valves, diastolic function matures through gestation and through the third week of postnatal life, with progressive improvements in active ventricular filling (57), similar to data found in other studies of mice and human $(56,58)$. Thus, UBM-Doppler appears well-suited as a tool to study embryonic, fetal, and postnatal developmental cardiovascular physiology.

Malformed hearts: pathophysiology of embryonic heart failure. More recently, UBM-Doppler has been applied to define in utero cardiovascular physiology when there is a malformed heart. The ability to provide detailed correlation of genotype with functional and structural phenotype is an exciting development in functional genomics. Given the uniqueness of the prenatal circulation (39), it is clear that characterization of cardiovascular failure in the developing mouse embryo will require imaging strategies that can dissect out the relative contributions of each critical component of the embryonic cardiovascular system, something that $7.5-15 \mathrm{MHz}$ clinical ultrasound systems cannot achieve.

We have recently studied the NFATCl-/- null mutant mouse (27), which lacks outflow tract (aortic and pulmonary) valves, and dies between E13.5 and 17.5 of presumed heart failure (59). We hypothesized that the absence of outflow tract valves would lead to abnormally reversed blood flow patterns, as blood regurgitated back into the embryonic heart through an unguarded outflow tract, and that such volume overload would lead to progressive systolic functional deterioration. While the hallmark reversed aortic flow was indeed revealed (Fig. 4), the mechanisms underlying cardiovascular failure turned out to be quite complex, comprising reduced cardiac output, diastolic dysfunction, and alterations in the distribution of blood flow between the embryo and placental bed;

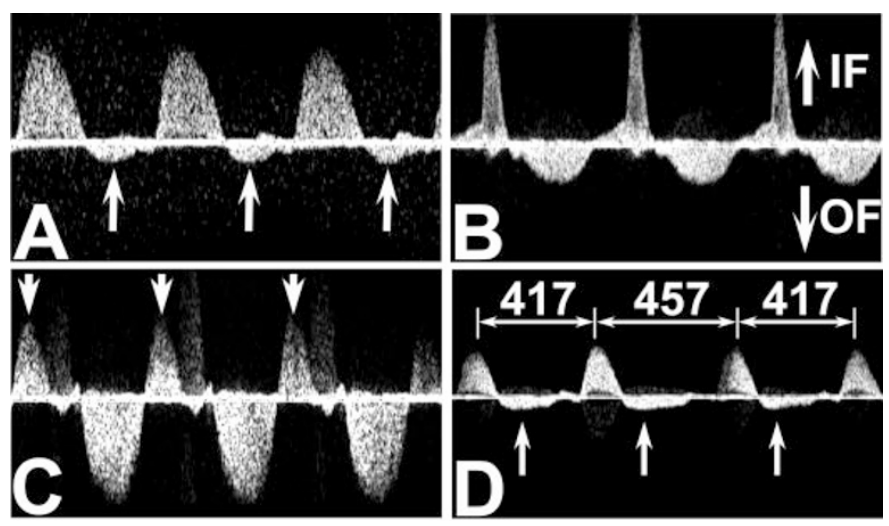

Figure 4. Abnormal physiology in an E13.5 NFATC1-/- null mutant mouse embryo. A) Reversed diastolic flow in the dorsal aorta (arrows), which is not present in normal embryos. B) Normal left ventricular inflow (IF)_outflow (OF) pattern in the same embryo, with the Doppler sample volume positioned somewhat away from the outflow tract. $C$ ) Abnormal reversed diastolic flow (arrows) in the proximal aorta, with the Doppler sample volume now in the outflow tract. D) Isolated arrhythmia in a different NFATC1-/- mouse embryo; varying cycle lengths are shown (in msec), even as reversed aortic flow is evident (arrows). chronic systolic dysfunction, curiously, was not a feature of this multifaceted heart failure (27).

In vivo cardiac morphology. The small size of individual structures (valves, myocardial wall, etc.) and their rapidity of motion (200-300 beats/min) have conspired to limit the amount of detailed in vivo morphologic data published to date. Nevertheless, beginning at E9.5, major events in cardiac morphogenesis have been visualized with UBM, such as cardiac looping; outflow tract septation (E11.5, E12.5); ventricular chamber septation (E12.5, E13.5), including the normal ventricular septal defect seen through $\sim$ E14 (25); and appearance of the atrio-ventricular valves (E13.5) (25,39; see Fig. 5). To date, however, at gestational stages $<15.5 \mathrm{~d}$, the internal anatomy of the embryonic heart is still difficult to ascertain, and no rigorous data have been published to date as to the consistency and reliability with which one can discern cardiac morphology in vivo.

Developmental vascular and blood imaging. Embryonic blood appears to act much like a Newtonian fluid, exhibiting laminar flow with a parabolic spatial velocity profile $(9,38)$. The intrinsic contrast offered by primitive nucleated erythroblasts at UBM frequencies may facilitate assessment of major vascular development (22). Arterial diameters and the presence of blood flow as indicated by a speckle motion are readily visualized from the earliest stages of cardiovascular development $(24,26,42)$. Ultrasonic contrast-enhanced imaging shows promise in improving the imaging of embryonic mouse vascular development (60).

Backscatter from blood depends somewhat on the shear rate (4), but also morphologic characteristics of blood cells, mainly erythrocytes. The major contributing factor to the echodensity of embryonic blood seems to be the presence of a dense, pyknotic nucleus, imaged by high UBM frequencies (54). An exciting advance in developmental hematology, one may in
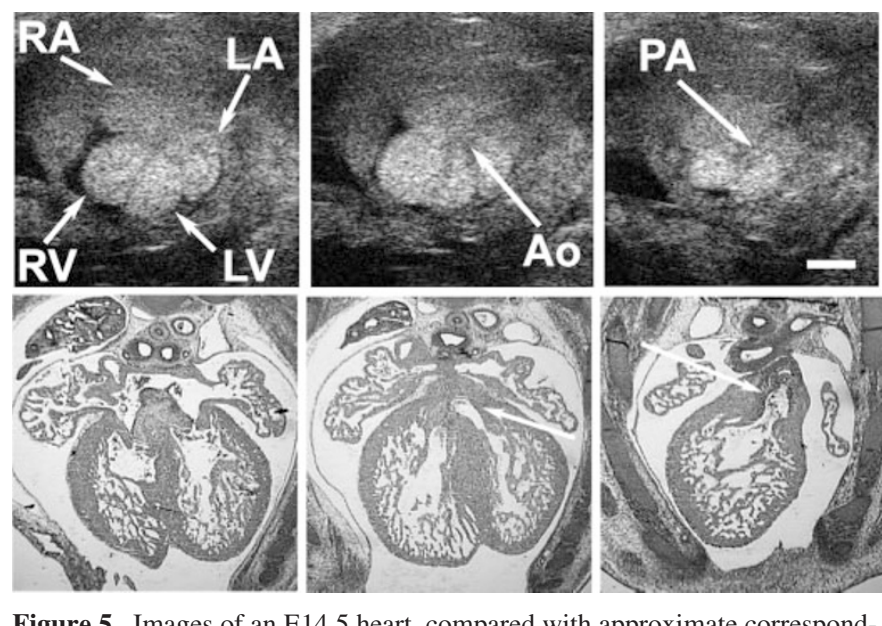

Figure 5. Images of an E14.5 heart, compared with approximate corresponding histologic sections from the same embryo (Note: there are mild differences in the rotation between UBM and histology). Left panel: Four-chamber view of the heart (LA, left atrium; LV, left ventricle; RA, right atrium; RV, right ventricle). The inter-ventricular septum and atrio-ventricular valves can be seen by UBM. Middle panel: with more cranial imaging, the proximal aorta (Ao) is seen arising from the LV, corresponding to the arrow on histology. Right panel: with further cranial imaging, the pulmonary artery (PA) can be seen arising from the RV, crossing the plane of the aorta in normal great arterial configuration. 
the future be able to follow certain characteristics of blood in a non-invasive manner throughout embryonic development.

In summary, UBM-Doppler possesses the ability to study numerous aspects of cardiovascular physiology in the in utero mouse embryo during critical stages of cardiovascular morphogenesis. Furthermore, the potential for in vivo morphologic definition suggests the ability to correlate living structure with function in the future.

\section{POTENTIAL FOR IMAGING OTHER ORGAN SYSTEMS}

Morphologic information has not been as forthcoming as functional cardiovascular data. However, several examples of UBM-Doppler imaging of non-cardiac organ systems will serve to illustrate its potential utility in extracting detailed data on developmental processes (Fig. 6).

A decade ago, Turnbull et al. (28) were the first to report the use of $50 \mathrm{MHz}$ UBM imaging of living, in utero mouse embryos. In Wnt-1 null mutant mice, they were able to distinguish mid-hindbrain deletions in E10.5-11.5 embryos; moreover, three-dimensional reconstruction of two-dimensional image datasets allowed quantitative comparisons between mutant and control embryos. Doppler flow in the developing brain has also been obtained, although no specific physiologic data have been published $(39,53)$. Our laboratory is now testing the potential for non-cardiac (e.g., neural tube) screens during primary assessment of cardiovascular defects (Phoon and Ji, unpublished observations; Fig. 7).

More recently, the overall processes from implantation through growth and placentation have been imaged in a qualitative manner $(22,25)$. Remarkably, the inner cell mass of the recently-implanted embryo could be visualized at E5.5, and by E7.5, the three cavities of the embryo demonstrated:
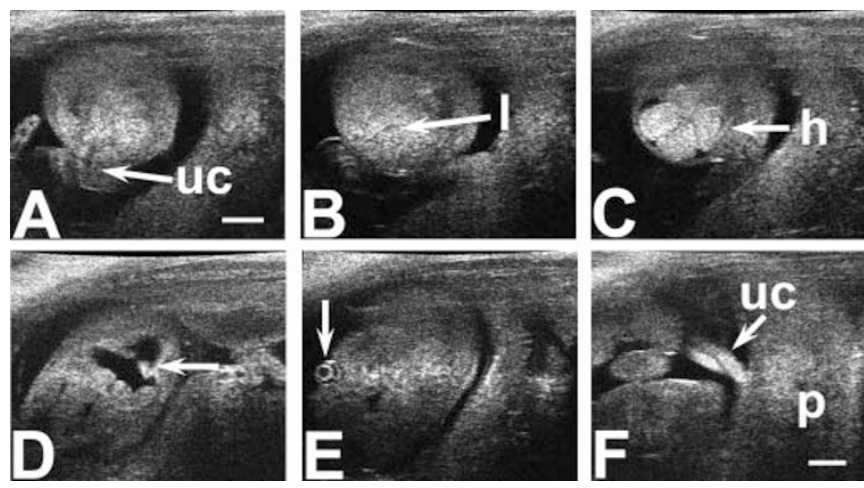

Figure 6. Serial UBM sections in the transverse or near-transverse plane in an in vivo E14.5 mouse embryo, obtained during a caudal to cranial sweep $(A-E)$, and additional image of umbilical cord inserting into placenta $(F) . A)$ Arising from the ventral abdominal wall, the umbilical cord (uc) can be seen. $B$ ) The liver (1) is seen as a homogeneous structure within the abdomen. $C$ ) The four chambers of the heart (h) can be seen, with the inter-ventricular and inter-atrial septa, and the atrio-ventricular valves. $D$ ) In the brain, cerebral fourth ventricles can be seen as echolucent structures; the arrow points to the choroid plexus. E) Arrow points to the embryonic eye. F) The umbilical cord (uc), consisting of an intertwined umbilical artery and umbilical vein, can be seen inserting into the placenta (p). Note the bright echogenic blood, producing natural contrast at these stages; no ultrasonic contrast agent was injected. Scale markers, $1 \mathrm{~mm}$.
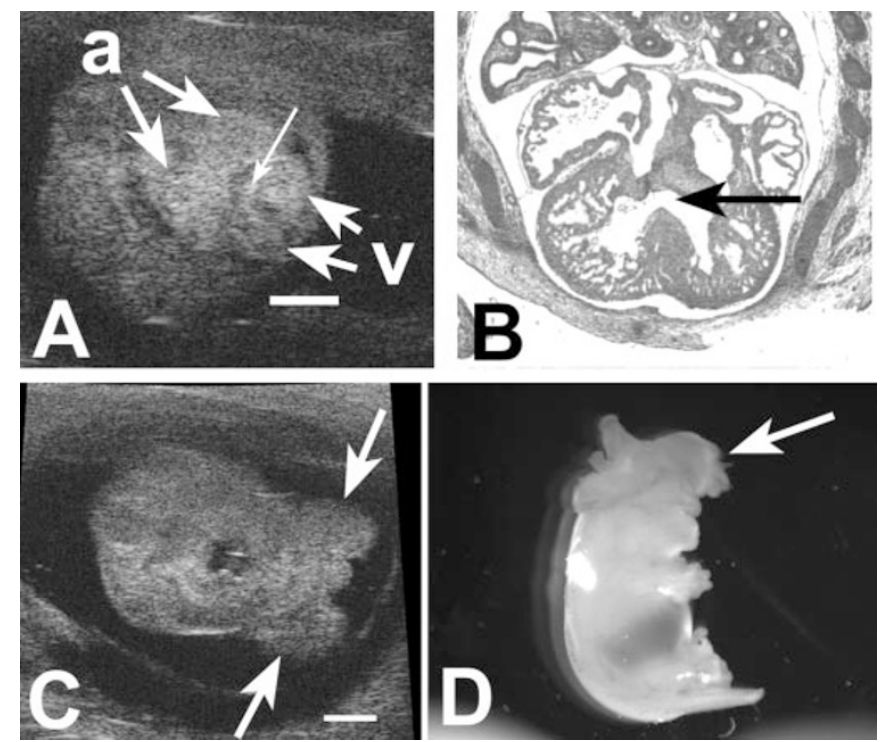

Figure 7. Examples of abnormal morphology at E13.5. A, B) An interventricular communication (small arrow) can be seen in both the UBM and histologic sections, respectively. Although an inter-ventricular communication can be normal at this stage, the image demonstrates the potential for diagnosing a true ventricular septal defect. $\mathrm{a}=$ atria, $\mathrm{v}=$ ventricles. $C, D$ : The UBM image and gross examination show exencephaly (arrows). Scale markers, $1 \mathrm{~mm}$.

the amniotic cavity, the coelomic cavity, and the ectoplacental cavity. The placenta could also be imaged from very early in gestation. Investigators have very recently begun to image placental development, with UBM able to discern normal calcifications particularly at the junction between the maternal decidua and the fetal trophoblast giant cells of the ecto-placental cone, along with normal developmental changes in these calcium hydroxyapatite deposits (61).

Investigators have also published UBM images of the limbs and spine of the developing mouse embryo (22); skin blister formation in the E12.5 to E13.5 GRIP1-/- null mutant
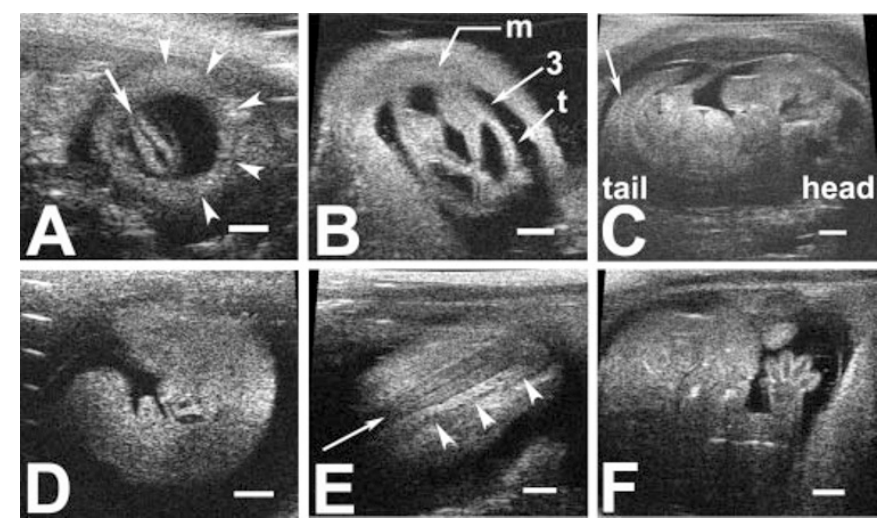

Figure 8. Examples of UBM imaging non-cardiac structures at various gestational stages. A) E10.5 open neural tube (arrow) of embryo encased within a muscular uterus (arrowheads); B) E12.5 brain, showing the mesencephalic vesicle ( $m$, future aqueduct), third ventricle (3), and telencephalic vesicle (t, future lateral ventricles); $C$ ) E13.5 embryo in a sagittal orientation, showing head and tail with spine seen clearly near the tail (arrow); D) dead E14.5 embryo in a sagittal orientation, showing homogeneity of tissues and an echolucent pericardial effusion; E) E15.5 spinal cord (arrow) and vertebral bodies (arrowheads); F) E15.5 head and forelimb. Scale markers, $1 \mathrm{~mm}$. 
Table 2. Studies of UBM image-guided microinjection into embryonic mouse brain

\begin{tabular}{|c|c|c|c|c|}
\hline Study & Injected Material & Location & Stage (E) & Purpose \\
\hline Olsson, 1997 (65) & Cells & Brain parenchyma & 13.5 & Engraftment \\
\hline Liu, 1998 (66) & Cells & Brain, limb & $9.5,10.5$ & Gain-of-function, ectopic Shh expression \\
\hline Gaiano, 1999 (67) & Retroviral vectors & Amniotic space & 8.5 & Gain-of-function, CNS infection \\
\hline McCarthy, 2001 (68) & Retroviral vectors & Lateral ventricles & 9.5 & Telencephalic cell lineage \\
\hline Wichterle, 2001 (69) & Labeled cells & LGE, MGE & 13.5 & Engraftment \\
\hline Weiner, 2002 (70) & Retroviral vectors & Cerebellum & 13.5 & Shh-activated medulloblastoma induction \\
\hline
\end{tabular}

CNS, central nervous system; LGE, lateral ganglionic eminence; MGE, medial ganglionic eminence; Shh, Sonic hedgehog.

In all of these experiments, survival rates post-injection were in the range of $50 \%$.

mouse (25); and maturational processes in the early postnatal mouse eye $(62,63)$. The remarkable resolution with which one can image non-cardiac structures such as the brain, spine, limbs, and eye strongly suggests an important role for UBM in helping to answer fundamental questions about embryonic patterning and development (Fig. 8).

\section{INTERVENTION AND MANIPULATION OF THE IN UTERO MOUSE}

Intervention via microinjection into the in utero mouse embryo/fetus is possible without special imaging, at later gestational stages (64). Given its good, in vivo definition of morphologic detail, UBM can facilitate the manipulation of the mouse embryo especially at younger stages, perturbing developmental processes to gain insight into such processes. Real-time, in utero imaging has made it possible to perform UBM image-guided injections of cells, viruses, or other agents into precise locations in the developing embryo at various developmental stages (Fig. 2). Following microinjections into specific sites within the embryonic mouse brain, for example, investigators have studied progenitor engraftment, ectopic gene expression with gain-of-function, cell lineages, and tumor induction (65-70; summarized in Table 2). It should be noted, however, that intra-embryonic injections challenge even the normal embryo and carry a substantial mortality rate of approximately $50 \%$. Therefore, caution must be exercised if one were to perform microinjections into mutant embryos, where survival is likely to be worse and survival itself may represent a selection bias.

Our laboratory has recently begun to assess the feasibility of pharmacological manipulation of the in utero mouse embryonic cardiovascular system. In preliminary experiments utilizing essentially the same approach as that pioneered by Turnbull and colleagues, we have obtained convincing hemodynamic responses to $\beta$-adrenergic agents (Phoon and Ji, unpublished data; Fig. 9).

In summary, UBM image-guided manipulation of the in utero mouse embryo facilitates genetic gain- or loss-offunction interventions, and manipulation of organ and cellular physiology in vivo, providing the developmental biologist with a powerful tool to gain insights into various developmental processes. Moreover, the testing of therapeutic strategies in the in utero mouse embryo may now be possible.

\section{FUTURE CHALLENGES}

Now proven to be an important phenotyping tool for embryonic mouse research, UBM-Doppler and similarly innova-
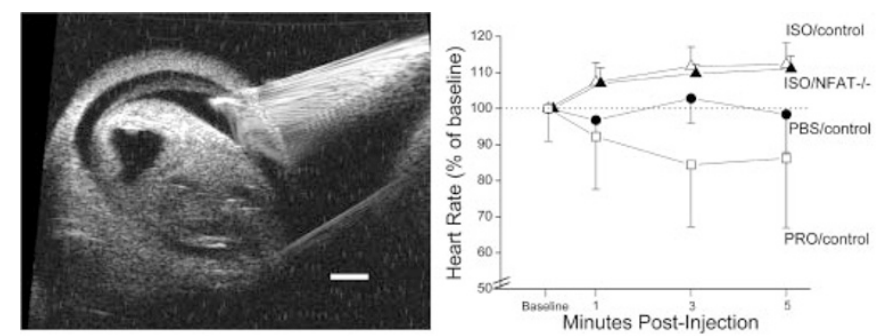

Figure 9. Feasibility of pharmacological manipulation of the in utero living mouse embryo. Left panel: UBM image-guided placement of the microinjection needle into the intraamniotic fluid space adjacent to an E13.5 embryo (scale marker, $1 \mathrm{~mm}$ ). Right panel: Heart rate responses in E13.5-14.5 embryos to $1-\mu \mathrm{L}$ injections of PBS into control embryos (PBS/control: solid circles); of $10 \mathrm{mM}$ isoproterenol into control embryos (ISO/control: open triangles); of $10 \mathrm{mM}$ isoproterenol into NFATC1-/- null mutant embryos (ISO/NFAT-/-: solid triangles); and of $10 \mathrm{mM}$ propranolol into control embryos (PRO/control: open squares). Isoproterenol increased heart rates in both control and NFATC1-/- embryos by an average of $\sim 10 \%$, whereas propranolol reduced heart rates by $\sim 15 \%$ in control embryos.

tive imaging can be used to answer important questions in developmental biology. In contrast to the gene-up strategy, our physiology-down approach begins with characterization of systems and organ physiology as the first step to unraveling cellular-level pathways and mechanisms. Future challenges include foremost the development of high-throughput assays and approaches. Advances in imaging approaches will by necessity be wedded to ongoing improvements in spatial and temporal resolution, imaging quality and image processing. Molecular imaging, an exciting development in ultrasound and $\mu$ MRI (71), may eventually allow UBM to elucidate metabolic and biochemical events at the cellular and molecular levels.

\section{REFERENCES}

1. Rossant J, McKerlie C 2001 Mouse-based phenogenomics for modelling human disease. Trends Mol Med 7:502-507

2. Gruber PJ, Epstein JA 2004 Development gone awry: congenital heart disease. Circ Res 94:273-283

3. Hoffman JI 1995 Incidence of congenital heart disease: II. Prenatal incidence. Pediatr Cardiol 16:155-165

4. Foster FS, Pavlin CJ, Harasiewicz KA, Christopher DA, Turnbull DH 2000 Advances in ultrasound biomicroscopy. Ultrasound Med Biol 26:1-27

5. Conway SJ, Kruzynska-Frejtag A, Kneer PL, Machnicki M, Koushik SV 2003 What cardiovascular defect does my prenatal mouse have, and why? Genesis 35:1-21

6. Rothenberg F, Efimov IR, Watanabe M 2004 Functional imaging of the embryonic pacemaking and cardiac conduction system over the past 150 years: technologies to overcome the challenges. Anat Rec A Discov Mol Cell Evol Biol 280:980-989

7. Keller BB, MacLennan MJ, Tinney JP, Yoshigi M 1996 In vivo assessment of embryonic cardiovascular dimensions and function in day-10.5 to -14.5 mouse embryos. Circ Res 1996;79:247-255

8. Jones EAV, Crotty D, Kulesa PM, Waters CW, Baron MH, Fraser SE, Dickinson ME 2002 Dynamic in vivo imaging of postimplantation mammalian embryos using whole embryo culture. Genesis 34:228-235

9. Jones EAV, Baron MH, Fraser SE, Dickinson ME 2004 Measuring hemodynamic changes during mammalian development. Am J Physiol Heart Circ Physiol 287:H1561-1569 
10. MacLennan MJ, Keller BB 1999 Umbilical arterial blood flow in the mouse embryo during development and following acutely increased heart rate. Ultrasound Med Biol 25:361-370

11. Ishiwata T, Nakazawa M, Pu WT, Tevosian SG, Izumo S 2003 Developmental changes in ventricular diastolic function correlate with changes in ventricular myoarchitecture in normal mouse embryos. Circ Res 93:857-865

12. Smith BR 2001 Magnetic resonance microscopy in cardiac development. Microsc Res Tech 52:323-330

13. Schneider JE, Bamforth SD, Farthing CR, Clarke K, Neubauer S, Bhattacharya S 2003 High-resolution imaging of normal anatomy, and neural and adrenal malformations in mouse embryos using magnetic resonance microscopy. J Anat 202:239247

14. Schneider JE, Bose J, Bamforth SD, Gruber AD, Broadbent C, Clarke K, Neubauer S, Lengeling A, Bhattacharya S 2004 Identification of cardiac malformations in mice lacking Ptdsr using a novel high-throughput magnetic resonance imaging technique. BMC Dev Biol 4:16

15. Nieman BJ, Bock NA, Bishop J, Chen XJ, Sled JG, Rossant J, Henkelman RM 2005 Magnetic resonance imaging for detection and analysis of mouse phenotypes. NMR Biomed 18:447-468

16. Hogers B, Gross D, Lehmann V, Zick K, De Groot HJM, Gittenberger-De Groot AC, Poelmann RE 2000 Magnetic resonance microscopy of mouse embryos in utero. Anat Rec 260:373-377

17. Chapon C, Franconi F, Roux J, Marescaux L, Le Jeune JJ, Lemaire L 2002 In utero time-course assessment of mouse embryo development using high resolution magnetic resonance imaging. Anat Embryol 206:131-137

18. Yelbuz TM, Choma MA, Thrane L, Kirby ML, Izatt JA 2002 Optical coherence tomography. A new high-resolution imaging technology to study cardiac development in chick embryos. Circulation 106:2771-2774

19. Sharpe J, Ahlgren U, Perry P, Hill B, Ross A, Hecksher-Sorenson J, Baldock R, Davidson D 2002 Optical projection tomography as a tool for 3D microscopy and gene expression studies. Science 296:541-545

20. Sharpe J 2004 Optical projection tomography. Annu Rev Biomed Eng 6:209-228

21. Coatney RW 2001 Ultrasound imaging: principles and applications in rodent research. ILAR J 42:233-247

22. Foster FS, Zhang MY, Zhou YQ, Liu G, Mehi J, Cherin E, Harasiewicz KA, Starkoski BG, Zan L, Knapik DA, Adamson SL 2002 A new ultrasound instrument for in vivo microimaging of mice. Ultrasound Med Biol 28:1165-1172

23. Gui YK, Linask KK, Khowsathit P, Huhta JC 1996 Doppler echocardiography of normal and abnormal embryonic mouse heart. Pediatr Res 40:633-642

24. Phoon CKL, Aristizabal O, Turnbull DH 200040 MHz Doppler characterization of umbilical and dorsal aortic blood flow in the early mouse embryo. Ultrasound Med Biol 26:1275-1283

25. Zhou YQ, Foster FS, Qu DW, Zhang M, Harasiewicz KA, Adamson SL 2002 Applications for multifrequency ultrasound biomicroscopy in mice from implantation to adulthood. Physiol Genomics 10:113-126

26. Ji RP, Phoon CKL, Aristizábal O, McGrath KE, Palis J, Turnbull DH 2003 Onset of cardiac function during early mouse embryogenesis coincides with entry of primitive erythroblasts into the embryo proper. Circ Res 92:133-135

27. Phoon CKL, Ji RP, Aristizábal O, Worrad DM, Zhou B, Baldwin HS, Turnbull DH 2004 Embryonic heart failure in NFATC1-/- mice: novel mechanistic insights from in utero ultrasound biomicroscopy. Circ Res 95:92-99

28. Turnbull DH, Bloomfield TS, Baldwin HS, Foster FS, Joyner AL 1995 Ultrasound backscatter microscope analysis of early mouse embryonic brain development. Proc Natl Acad Sci 92:2239-2243

29. Brown AS, Reid AD, Leamen L, Cucevic V, Foster FS 2004 Biological effects of high-frequency ultrasound exposure during mouse organogenesis. Ultrasound Med Biol 30:1223-1232

30. Huang GY, Wessels A, Smith BR, Linask KK, Ewart JL, Lo CW 1998 Alteration in connexin 43 gap junction gene dosage impairs conotruncal heart development. Dev Biol 198:32-44

31. Mäki JM, Räsänen J, Tikkanen H, Sormunen R, Mäkikallio K, Kivirikko KI, Soininen R 2002 Inactivation of lysyl oxidase gene lox leads to aortic aneurysms, cardiovascular dysfunction, and perinatal death in mice. Circulation 106:2503-2509

32. Mäkikallio K, Rounioja S, Vuolteenaho O, Paakkari J, Hallman M, Räsänen J 2006 2006 Fetal cardiac natriuretic peptide expression and cardiovascular hemodynamics in endotoxin-induced acute cardiac dysfunction in mouse. Pediatr Res 59:180-184

33. Chang CP, Chen L, Crabtree GR 2003 Sonographic staging of the developmental status of mouse embryos in utero. Genesis 36:7-11

34. Spurney CF, Leatherbury L, Lo CW 2004 High-frequency ultrasound database profiling growth, development, and cardiovascular function in $\mathrm{C} 57 \mathrm{BL} / 6 \mathrm{~J}$ mouse fetuses. J Am Soc Echocardiogr 17:893-900

35. Yu Q, Shen Y, Chatterjee B, Siegfried BH, Leatherbury L, Rosenthal J, Lucas JF, Wessels A, Spurney CF, Wu YJ, Kirby ML, Svenson K, Lo CW 2004 ENU induced mutations causing congenital cardiovascular anomalies. Development 131:62116223

36. Shen Y, Leatherbury L, Rosenthal J, Yu Q, Pappas MA, Wessels A, Lucas J, Siegfried B, Chatterjee B, Svenson K, Lo CW 2005 Cardiovascular phenotyping of fetal mice by non-invasive high-frequency ultrasound studies facilitates recovery of ENU-induced mutations causing congenital cardiac and extracardiac defects. Physiol Genomics 24:23-26

37. Leatherbury L, Yu Q, Lo CW 2003 Non-invasive phenotypic analysis of cardiovascular structure and function in fetal mice using ultrasound. Birth Defects Res C Embryo Today 69:83-91

38. Phoon CKL, Aristizábal O, Turnbull DH 2002 Spatial velocity profile in mouse embryonic aorta and Doppler-derived volumetric flow: a preliminary model. Am J Physiol Heart Circ Physiol 283:H908-H916
39. Phoon CKL, Turnbull DH 2003 Ultrasound biomicroscopy-Doppler in mouse cardiovascular development. Physiol Genomics 14:3-15

40. Goertz DE, Yu JL, Kerbel RS, Burns PN, Foster FS 2003 High-frequency 3-D color-flow imaging of the microcirculation. Ultrasound Med Biol 29:39-51

41. Ketterling JA, Aristizábal O, Turnbull DH, Lizzi FL 2005 Design and fabrication of a $40-\mathrm{MHz}$ annular array transducer. IEEE Trans Ultrason Ferroelectr Freq Control 52:672-681

42. Srinivasan S, Baldwin HS, Aristizábal O, Kwee L, Labow M, Artman M, Turnbull DH 1998 Non-invasive, in utero imaging of mouse embryonic development with 40-MHz echocardiography. Circulation 98:912-918

43. Ji RP, Phoon CK 2005 Non-invasive localization of mouse embryos by ultrasound biomicroscopy (UBM)-Doppler allows genotype-phenotype correlation. J Am Soc Echocardiogr 18:1415-21

44. Furukawa S, MacLennan MJ, Keller BB 1998 Hemodynamic response to anesthesia in pregnant and nonpregnant ICR mice. Lab Anim Sci 48:357-363

45. Roth DM, Swaney JS, Dalton ND, Gilpin EA, Ross J Jr 2002 Impact of anesthesia on cardiac function during echocardiography in mice. Am J Physiol Heart Circ Physiol 282:H2134-H2140.

46. Janssen BJ, De Celle T, Debets JJ, Brouns AE, Callahan MF, Smith TL 2004 Effects of anesthetics on systemic hemodynamics in mice. Am J Physiol Heart Circ Physiol 287:H1618-H1624

47. Huang GY, Linask KK 1998 Doppler echocardiographic analysis of effects of tribromoethanol anesthesia on cardiac function in the mouse embryo: a comparison with pentobarbital. Lab Anim Sci 48:206-9

48. Mazze RI 1985 Fertility, reproduction, and postnatal survival in mice chronically exposed to isoflurane. Anesthesiology 63:663-667

49. Mazze RI, Wilson AI, Rice SA, Baden JM 1985 Fetal development in mice exposed to isoflurane. Teratology 32:339-345

50. Hande MP, Devi PU 1993 Effect of in utero exposure to diagnostic ultrasound on the postnatal survival and growth of mouse. Teratology 48:405-411

51. Warren JR, Shaw B, Steinkampf MP 1992 Inhibition of preimplantation mouse embryo development by isoflurane. Am J Obstet Gynecol 166:693-698

52. Nakazawa M, Clark EB, Hu N, Wispe J 1985 Effect of environmental hypothermia on vitelline artery blood pressure and vascular resistance in the stage 18, 21, and 24 chick embryo. Pediatr Res 19:651-654

53. Turnbull DH 1999 In utero ultrasound backscatter microscopy of early stage mouse embryos. Comput Med Imaging Graph 23:25-31

54. Le Floc'h J, Chérin E, Zhang MY, Akirav C, Adamson SL, Vray D, Foster FS 2004 Developmental changes in integrated ultrasound backscatter from embryonic blood in vivo in mice at high US frequency. Ultrasound Med Biol 30:1307-1319

55. Burggren WW 2004 What is the purpose of the embryonic heart beat? or how facts can ultimately prevail over physiological dogma. Physiol Biochem Zool 77:333-345

56. Phoon CKL 2001 Circulatory physiology in the developing embryo. Curr Opin Pediatr 13:456-464

57. Zhou YQ, Foster FS, Parkes R, Adamson SL 2003 Developmental changes in left and right ventricular diastolic filling patterns in mice. Am J Physiol Heart Circ Physiol 285:H1563-H1575

58. Mäkikallio K, Jouppila P, Räsänen J 2005 Human fetal cardiac function during the first trimester of pregnancy. Heart 91:334-338

59. Ranger AM, Grusby MJ, Hodge MR, Gravallese EM, de la Brousse FC, Hoey T, Mickanin C, Baldwin HS, Glimcher LH 1998 The transcription factor NF-ATc is essential for cardiac valve formation. Nature 392:186-190

60. Turnbull DH, Aristizábal O, Ketterling JA 2005 In vivo contrast-enhanced imaging of mouse vascular development. Proc IEEE Trans Ultrason Ferroelectr Freq Control Symp (in press)

61. Akirav C, Lu Y, Mu J, Qu DW, Zhou YQ, Slevin J, Holmyard D, Foster FS, Adamson SL 2005 Ultrasonic detection and developmental changes in calcification of the placenta during normal pregnancy in mice. Placenta 26:129-137

62. Brown AS, Zhang M, Cucevic V, Pavlin CJ, Foster FS 2005 In vivo assessment of postnatal murine ocular development by ultrasound biomicroscopy. Curr Eye Res 30:45-51

63. Brown AS, Leamen L, Cucevic V, Foster FS 2005 Quantitation of hemodynamic function during developmental vascular regression in the mouse eye. Invest Ophthalmol Vis Sci 46:2231-2237

64. Mitchell M, Jerebtsova M, Batshaw ML, Newman K, Ye X 2000 Long-term gene transfer to mouse fetuses with recombinant adenoviruses and adeno-associated virus (AAV) vectors. Gene Therapy 7:1986-1992

65. Olsson M, Campbell K, Turnbull DH 1997 Specification of mouse telencephalic and mid-hindbrain progenitors following heterotopic ultrasound-guided embryonic transplantation. Neuron 19:761-772

66. Liu A, Joyner AL, Turnbull DH 1998 Alternation of limb and brain patterning in early mouse embryos by ultrasound-guided injection of Shh-expressing cells. Mech Dev 75:107-115

67. Gaiano N, Kohtz JD, Turnbull DH, Fishell G 1999 A method for rapid gain-offunction studies in the mouse embryonic nervous system. Nat Neurosci $2 \cdot 812-819$

68. McCarthy M, Turnbull DH, Walsh CA, Fishell G 2001 Telencephalic neural progenitors appear to be restricted to regional and glial fates before onset of neurogenesis. J Neurosci 21:6772-6781

69. Wichterle H, Turnbull DH, Nery S, Fishell G, Alvarez-Buylla A 2001 In utero fate mapping reveals distinct migratory pathways and fates of neurons in the mammalian basal forebrain. Development 128:3759-3771

70. Weiner HL, Bakst R, Hurlbert MS, Ruggiero J, Ahn E, Lee WS, Stephen D, Zagzag D, Joyner AL, Turnbull DH 2002 Induction of medulloblastomas in mice by sonic hedgehog, independent of Gli1. Cancer Res 62:6385-6389

71. Liang HD, Blomley MJ 2003 The role of ultrasound in molecular imaging. Br J Radiol 76:S140-S150 